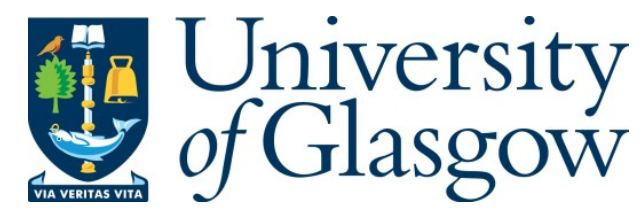

Witte, C., K remer, C., Cooper, J.M., and N eale, S.L. (2013) C ontinuous cell Iysis in microfluidics through acoustic and optoelectronic tweezers. In: SPIE Photonics W est M OEM S-M EM S, 2-7th Feb, San Francisco.

Copyright $\odot 2013$ Society of Photo Optical Instrumentation Engineers

A copy can be downloaded for personal non-commercial research or study, without prior permission or charge

Content must not be changed in any way or reproduced in any format or medium without the formal permission of the copyright holder(s)

When referring to this work, full bibliographic details must be given

$\underline{\text { http://eprints.gla.ac.uk/68041/ }}$

Deposited on: 30 J anuary 2014

Enlighten - Research publications by members of the U niversity of Glasgow http://eprints.gla.ac.uk 


\title{
Continuous cell lysis in microfluidics through acoustic and optoelectronic tweezers
}

\author{
Christian Witte*, Clemens Kremer, Jonathan M. Cooper, Steven L. Neale \\ University of Glasgow, Biomedical Engineering, Glasgow, G12 8LT, United Kingdom
}

\begin{abstract}
A versatile platform for efficient cell lysis using a combination of acoustic and electric fields in a microchannel is presented. Cell membrane disruption is triggered by electric fields inducing electroporation and then lysis. The principle of optoelectronic tweezers (OET) is applied to control the electric field strength and a surface acoustic wave transducer is attached to an OET chip to implement acoustic tweezing (AT). The system is characterized in terms of spatial control of electric fields, single cell precision and lysis times. Under continuous operation, a combination of AT and OET improves cell lysis significantly achieving for sample concentrations of $10^{6}$ cell $/ \mathrm{ml} \mathrm{lysis} \mathrm{efficiencies} \mathrm{of}>99 \%$.
\end{abstract}

Keywords: cell lysis, micromanipulation, optoelectronic tweezer, acoustic tweezer, microfluidics, blood

\section{INTRODUCTION}

Cell lysis is an important step in bioanalytical systems. It describes the break down of the impermeable cell membrane and the release of intracellular macromolecules and organelles. The resulting cell lysate can be analysed towards the concentration or presence of certain proteins, DNA or RNA. Specificity and selectivity of the method are often of interest when individual analysis or the enrichment of cells are necessary.

In the last decade several strategies based on chemical, optical, acoustical and electrical methods were developed to integrate lysis in microfluidic environments ${ }^{1,2}$. Here, one of the challenges is to provide a flexible system capable to perform fast single cell lysis as well as high-throughput and continuous procedures. While chemical lysis is well suited to massively lyse cells, it is difficult to get control on single cell level. Furthermore, chemicals might interfere with important analytes in the lysate. Individual cell treatment in a population is a matter of focussing an external force precisely onto a desired cell. Here, conventional electrical methods based on solid electrodes of micrometer dimensions or microchannel restriction zones can be used but rely heavily on cell-to-electrode position (batch mode) or are limited to flow-through applications. A precise spatial and flexible control of forces for single cell lysis can be achieved using optical methods. For instance, highly-focused pulsed laser microbeams are able to provide very fast $(\sim \mu \mathrm{s})$ lysis times ${ }^{3}$. Such a system is especially useful for studying cellular analytes with rapid reaction kinetics. However, limitations can occur when dense populations are investigated, as the laser-induced shockwave might influence neighbouring cells. Similiar restriction can be observed when using acoustic approaches. The creation of cavitation bubbles by localized high pressure points is difficult to apply on a single cell within a group of cells. Moreover, heat generation by absorption of acoustic energy can influence intracellular molecules (e.g. by denaturation) ${ }^{1}$.

This work presents a strategy were the flexibility of optical methods is combined with individual and efficient cell lysis capability of electrical methods. We use an optoelectronic tweezer (OET) chip ${ }^{4}$ to apply localized electric fields by illumination of a photoconductor and demonstrate the ability for single cell lysis as well as lysis of high cell concentrations. The setup can be combined with acoustic tweezing (AT) using an ultrasound transducer to produce a pressure distribution in the liquid. This leads to a rapid and localized concentration of cells along the microchannel chip and reduces the illumination area to induce lysis under continuous flow. The advantages of the system includes performing of experiments in batch and flow-through format. It can be applied to single cells and dense cell population and it offers the potential to integrate downstream analysis steps. 


\section{EXPERIMENTAL}

The transmembrane potential of a cell under physiological conditions is approximately $\sim 60 \mathrm{mV}$. When cells are exposed to strong electric fields $(\sim \mathrm{kV} / \mathrm{cm})$ electroporation and dielectric breakdown of the lipid bilayer can occur. Once the electric field exceeds a cell specific threshold, a compression pressure induces pore formation in the membrane. A further increase in the field strength leads to a chemical modification of the membrane by ionization and a breakdown, eventually. While electroporation can be reversible and useful for transfection experiments, a dielectric breakdown destroys the cell. The average transmembrane potential $\Delta \varphi$ to induce lysis is $\sim 1.1 \mathrm{~V}$ (figure $1 \mathrm{~A})^{2}$.

(A)

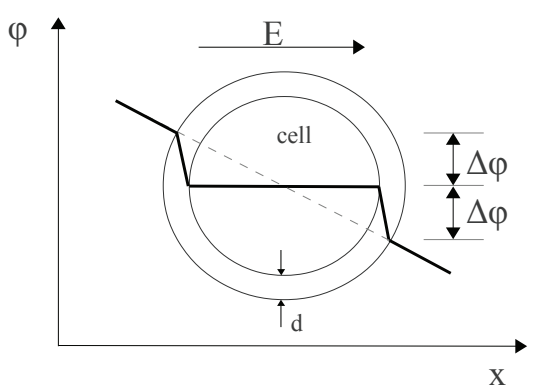

(B)

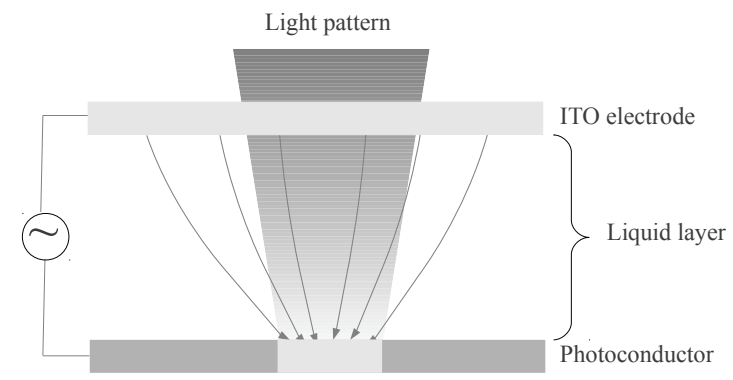

Figure 1. (A) Schematic of transmembrane potential $\Delta \varphi$ induced by an external electric field. An average voltage drop across the membrane of $\sim 1.1 \mathrm{~V}$ induces electroporation. (B) Schematic of the optoelectronic tweezer (OET) concept. AC voltage is applied between a photoconductor and an ITO electrode. Illumination generates a nonuniform electric field in the liquid.

Permanent or pulsed DC fields can be used for electrical lysis, however, in this work nonuniform AC fields are applied using the concept of optoelectronic tweezers (OET, figure 1B). Here, the electric field is the result of local changes in the conductivity of a photoconductor (amorphous silicon, a-Si) induced by selective illumination. When the light is switched off, most of the voltage drops across this photoconductor. But when illuminated, the conductivity increases by 2 orders of magnitudes which switches the voltage drop across the liquid layer. The light intensity to fully activate the photoconductor as well as the gap between the bottom and the top electrode influence the electric field strength in the liquid. In this work the optical intensity is in the range of several $\mathrm{W} / \mathrm{cm}^{2}$. The gap between the indium tin oxide glass electrode (ITO) and the photoconductor is $35 \mu \mathrm{m}$. The OET chip is made by structuring SU8 sandwiched between the electrodes (figure 2B) . $^{4}$ The selective illumination of the electrode is achieved using a DELL data projector connected to a PC running an imaging software (figure $2 \mathrm{~A}$ ). 

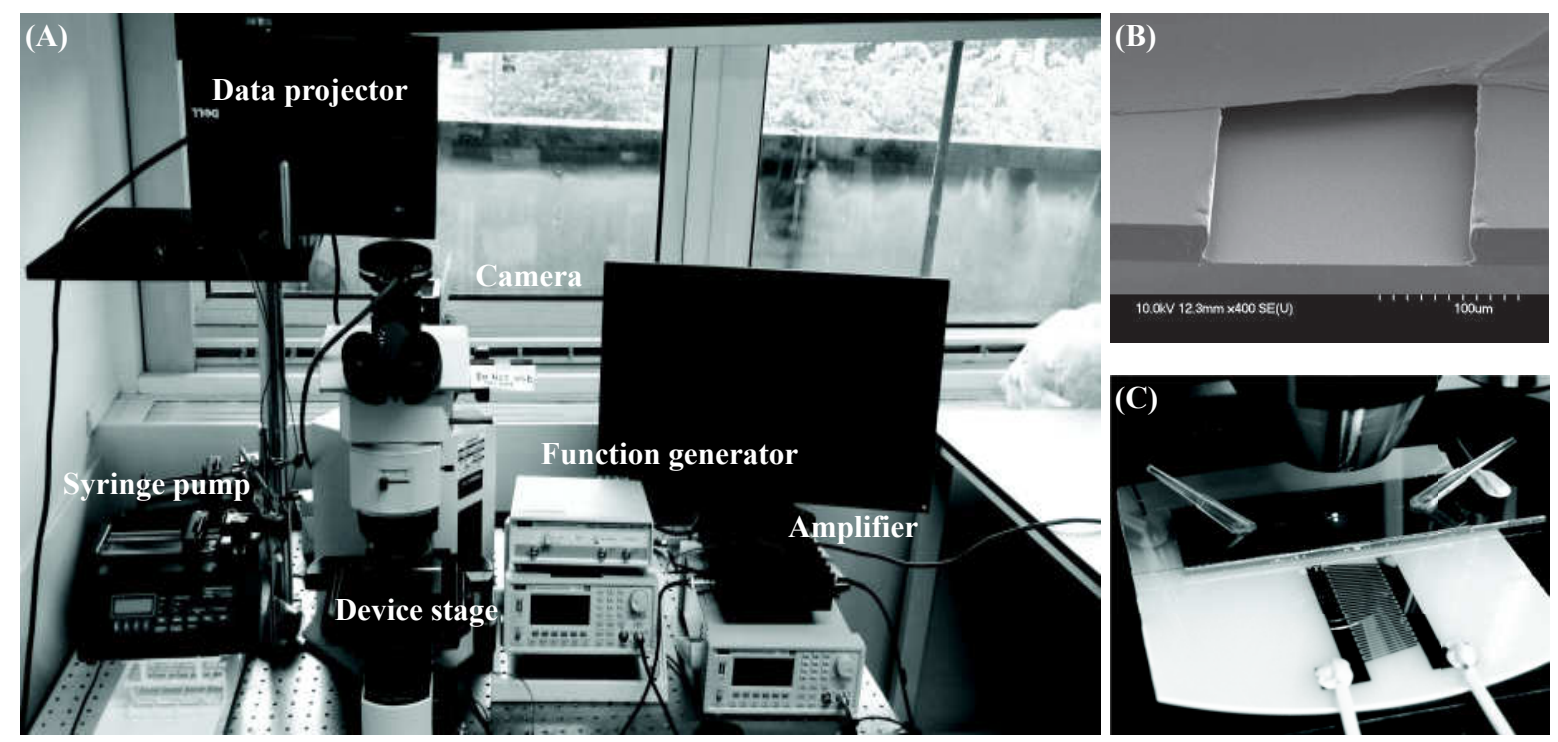

Figure 2. (A) Setup for optoelectronic and acoustic tweezing. (B) Example of an OET chip showing microchannel made from SU8 sandwiched between the photoconducting substrate (bottom) and the ITO electrode on top. (C) Assembled system, placed under the objective, containing the surface acoustic wave device for AT and the OET chip.

The OET chip is connected to a function generator where a $25 \mathrm{kHz}$ signal with an amplitude of $20 \mathrm{~V}$ (peak-to-peak) is applied between the photoconductor and the ITO electrode. A cell sample can be injected via the pre-drilled holes in the ITO substrate and the device can be used either in batch mode or when connected to a syringe pump in a continuous fashion. Furthermore, the chip, as independent unit, can be combined with an acoustic transducer to perform AT. Therefore, the OET chip is simply placed on top of the piezoelectric $\left(\mathrm{LiNbO}_{3}\right.$ ) substrate (figure 2C). The ultrasound is generated by a slanted interdigitated transducer ${ }^{4}$ and coupled to the OET chip. The frequency can be tuned within the range of $3 \mathrm{MHz}$ to $6 \mathrm{MHz}$ to match the resonance frequency of the OET chip. The channel width used in this work is $150 \mu \mathrm{m}$. A suitable resonance point is obtained at $3.96 \mathrm{MHz}$. At this particular frequency a standing wave with a characteristic pressure distribution of half a wavelength is observed (figure 3A). For instance, in figure 3B, the whole channel is occupied by blood cells. When the chip is actuated the cells undergo acoustophoretic movement towards the pressure node driven by the acoustic radiation force (figure 3C). This leads to a concentration as well as controlled localization of the cells. Prior to the lysis experiments, cells are diluted in DEP buffer (20 mS/m, pH 7, 310 mosmol/1).

(A)

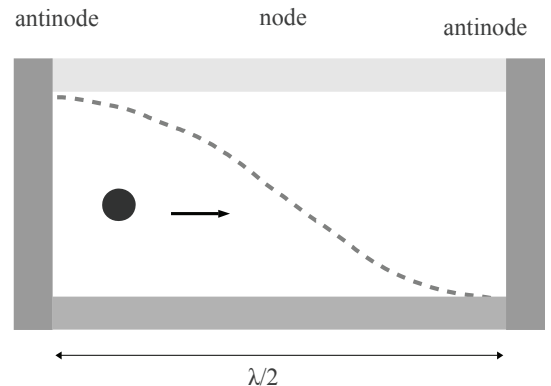

(B)

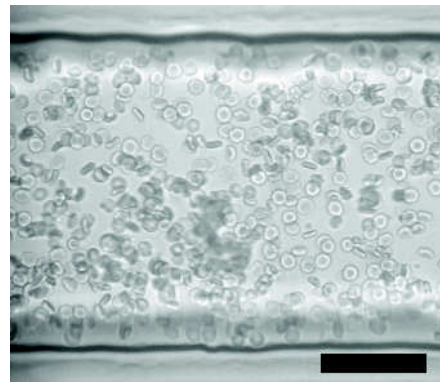

(C)

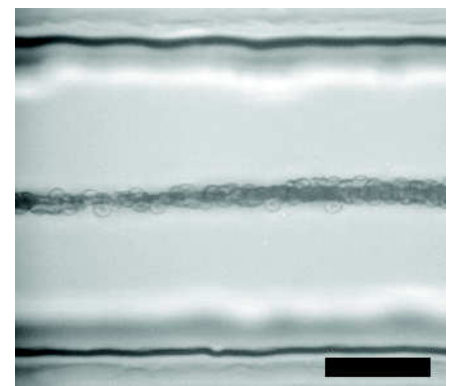

Figure 3. (A) Coupling of an acoustic wave results in characteristic pressure distribution in the microchannel under resonance condition. (B) Blood cells in a microchannel without acoustic actuation. (C) Actuation induces an acoustic radiation force which drives cells towards the pressure node. Scale bar: $50 \mu \mathrm{m}$. 


\section{RESULTS AND DISCUSSION}

The ability to dynamically pattern electric fields is the key to perform electroporation on single cell level. Using the OET system, light spots of a few micrometer can be defined which generate highly localized nonuniform electric fields. The position of the spot is controlled by an imaging software and can be used to target certain cells in a population. An example of this procedure is demonstrated in figure 4A-C. The experiment is performed in batch mode without AT. The red blood cells are first injected into the chip. After settling, cells of interest are selected and a light spot size ( $3 \mu \mathrm{m})$ is defined (figure 4A). The spot is moved and pointed directly at the cell, followed by an $1 \mathrm{~s} \mathrm{AC} \mathrm{signal}(20 \mathrm{~V}, 25 \mathrm{kHz})$. From figure 4B it can be seen that the cell contrast changes. The electric field strength is high enough to induce electroporation. The cellular content diffuses out and the cell loses contrast until it is almost undistinguishable from the background. While two cells are successfully lysed, neighbouring cell in submicron distance are unaffected by this selective process.
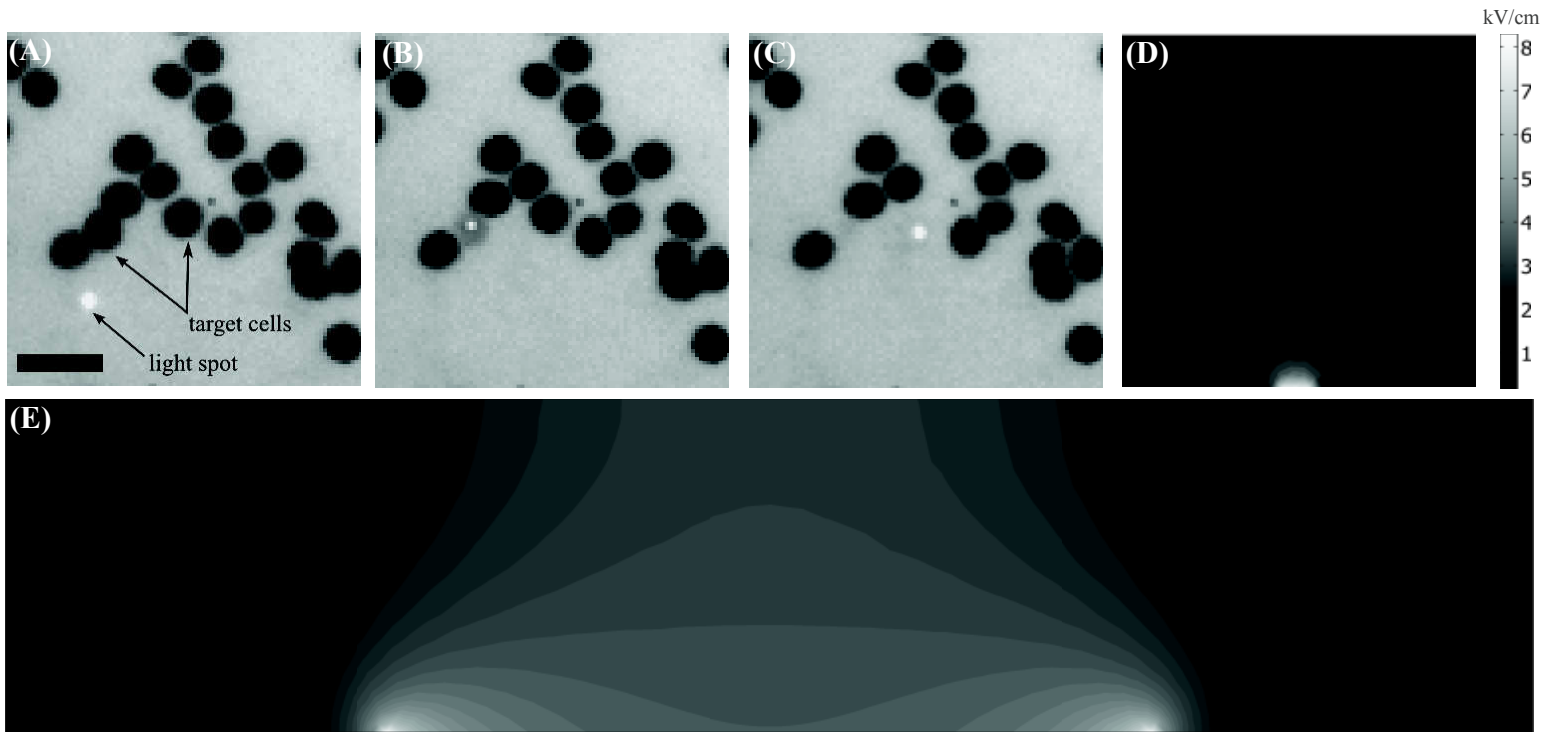

Figure 4. Selective lysing of red blood cells through light spot size and position control. (A) Target cells within a population and spot size is shown. The contrast of the image is altered to improve sharpness. Scale bar: $20 \mu \mathrm{m}$. (B) Chosen cell is undergoing lysis. (C) Target cells are lysed without affecting neighbouring cells. (D) Simulation of electric field $(\mathrm{kV} / \mathrm{cm}$ ) for a $3 \mu \mathrm{m}$ light spot in a $35 \mu \mathrm{m}$ height channel at $25 \mathrm{kHz}$ and $20 \mathrm{~V}$. (E) Simulation of electric field for a $80 \mu \mathrm{m}$ light spot in a $35 \mu \mathrm{m}$ height channel at $25 \mathrm{kHz}$ and $20 \mathrm{~V}$.

The electric field distribution of the illuminated OET chip and the potential across the cell membrane is investigated with a finite-element model implemented in COMSOL Multiphysics. The parameters used for the simulation are listed in table 1. The conductivity of a-Si is proportional to the optical intensity. The measured intensity in our systems is $7.35 \mathrm{~W} / \mathrm{cm}^{2}$ which gives a peak conductivity of $1.4 \mathrm{mS} / \mathrm{m}$. The intensity of the light pattern is considered to have a Gaussian profile. The dielectric properties of the cell membrane are from reference 5 . 
Table 1. Parameters used in this study

\begin{tabular}{llll}
\hline Channel cross section W x H & $160 \mu \mathrm{m} \times 35 \mu \mathrm{m}$ & Conductivity of a-Si (light state) & $1.4 \cdot 10^{-3} \mathrm{~S} / \mathrm{m}$ \\
Frequency (OET) & $25 \mathrm{kHz}$ & Permitivity $\varepsilon_{\mathrm{r}}$ of a-Si & 14 \\
Amplitude (OET) & $20 \mathrm{~V}$ & Cell membrane thickness & $7 \mathrm{~nm}$ \\
Conductivity of DEP buffer & $0.02 \mathrm{~S} / \mathrm{m}$ & Conductivity of cell membrane & $1 \cdot 10^{-6} \mathrm{~S} / \mathrm{m}$ \\
Permitivity $\varepsilon_{\mathrm{r}}$ of DEP Buffer & 79 & Permitivity $\varepsilon_{\mathrm{r}}$ of cell membrane & 12.5 \\
Thickness amorphous silicon (a-Si) & $1.5 \mu \mathrm{m}$ & Conductivity of cytosol & $0.8 \mathrm{~S} / \mathrm{m}$ \\
Conductivity a-Si (dark state) & $1.8 \cdot 10^{-5} \mathrm{~S} / \mathrm{m}$ & Permitivity $\varepsilon_{\mathrm{r}}$ of cytosol & 60 \\
\hline
\end{tabular}

The simulation of the electric field for a $3 \mu \mathrm{m}$ light spot (like it is used in figure 4A-C) is shown in figure 4D. Under the above mentioned conditions, results for the electric field strength show values of up to $8 \mathrm{kV} / \mathrm{cm}$. Due to the size of the spot, the high field area is well defined close to the surface of the photoconductor. In lateral and vertical direction the field strength decays very sharp. This result is of significance as it explains the area-defined selectivity where lysis is induced. In the presence of a red blood cell, the calculated voltage drop across the membrane is dependent on the lateral and vertical position relative to the light spot. For instance, a cell positioned above the light spot with a vertical distance of $1 \mu \mathrm{m}$ is experiencing a transmembrane voltage of $1.90 \mathrm{~V}$ across the membrane closest to the surface of the photoconductor. However, increasing the gap to $8 \mu \mathrm{m}$ results in a voltage drop of $0.53 \mathrm{~V}$ which is not sufficient for lysis. Based on the simulation results, a maximal displacement of $3 \mu \mathrm{m}$ in vertical direction is possible to achieve irreversible electroporation $(1.1 \mathrm{~V})$. The decay in the lateral electric field strength is stronger and a distance of $1 \mu \mathrm{m}$ from the light spot edge decreases the membrane potential to $0.79 \mathrm{~V}$ for a the vertical distance of $1 \mu \mathrm{m}$. A minimum gap of $0.5 \mu \mathrm{m}$ is necessary to overcome the threshold of $1.1 \mathrm{~V}$. The results agree very well with the experimental observation. Levitation of a cell in the chip impedes electroporation. One way to circumvent this limitation is the reduction of the channel thickness. Another approach but at the cost of single cell precision is the increase of the light spot. In figure 4E, the electric field for a light spot of $80 \mu \mathrm{m}$ is shown. The field strength is not increased but the broadened illumination leads to values of several $\mathrm{kV} / \mathrm{cm}$ across the whole channel height. The change in the light spot size enhances the electron-hole pair formation in the photoconductor and causes an improved voltage drop across the liquid layer ${ }^{6}$. Under these conditions a cell is undergoing lysis even when the distance is large (e.g. $30 \mu \mathrm{m}$, transmembrane potential of $1.3 \mathrm{~V})$.

In addition to controlling lysis on single cell level, the time it takes to irreversible damage the cell is of importance when working under continuous flow for high throughput applications. It determines the flow rates in the channel and influences subsequent analysis steps. To get an insight into the time scale to trigger lysis, experiments are conducted in batch mode which simplifies the observation over time compared to flow-through conditions. The system is characterised by varying the light exposure times. In figure 5A, a sequence of red bloods cells, treated with a $35 \mathrm{~ms}$ pulse, is shown. The typical biconcave shape of the cell can be observed before the light exposure. However, $10 \mathrm{~ms}$ after the pulse the cells undergo a substantial deformation. In the time frame from $10 \mathrm{~ms}$ to $2 \mathrm{~s}$, a transition from a biconcave morphology to a spherical one is observed. This is explained by a irreversible pore formation which leads to an uncontrolled flow of water across the membrane into the cell. The structural integrity is broken and the cells expand followed by diffusion of cellular content out of the cell. After tens of seconds only hollow membrane vesicles are left. A water influx can also lead to a break of the membrane. In figure 5B lymphocytes are treated with a $35 \mathrm{~ms}$ pulse. In less than $2 \mathrm{~s}$ after the exposure, the expanded cells burst open and release cell organelles and molecules. This differs significantly in the time scale and its way from red blood cells. The reason for this observation could be explained based on the function of the particular cell type. For instance, red blood cells experiencing high shear stresses, especially when squeezing through small capillaries in the body. Therefore the membrane contains a specialised protein composition which is characterised by flexibility and durability. 
(A)
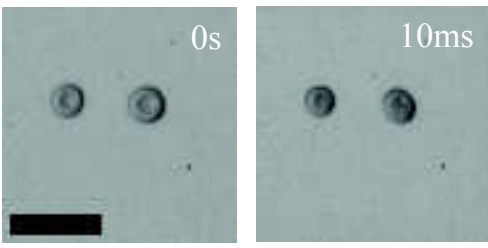

(B)
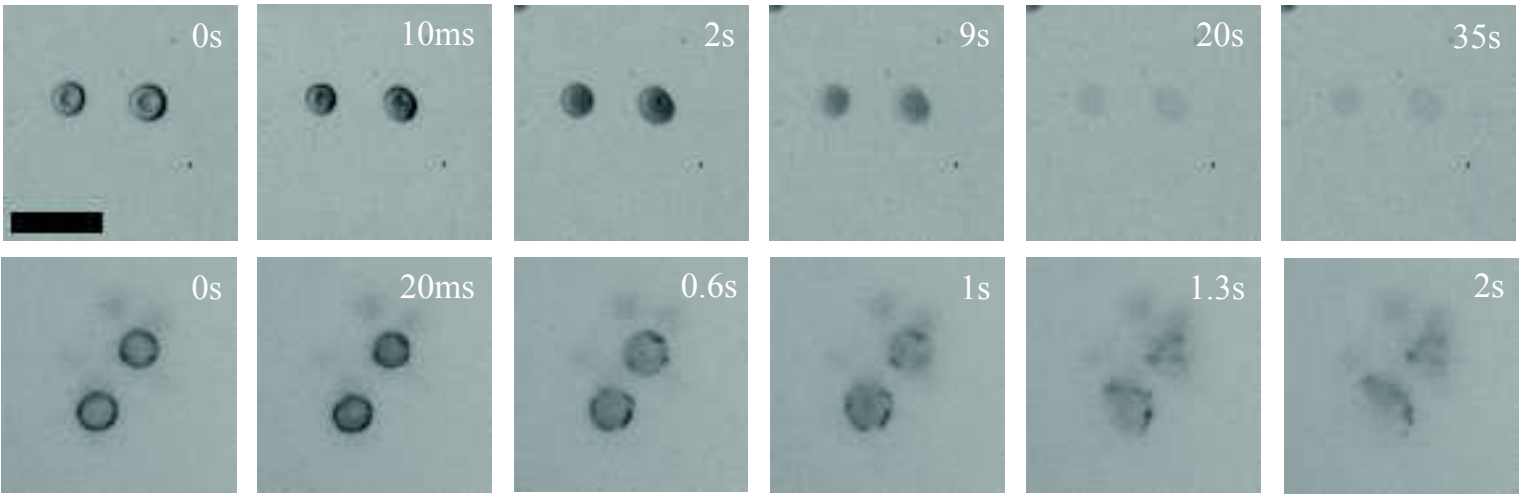

(C)

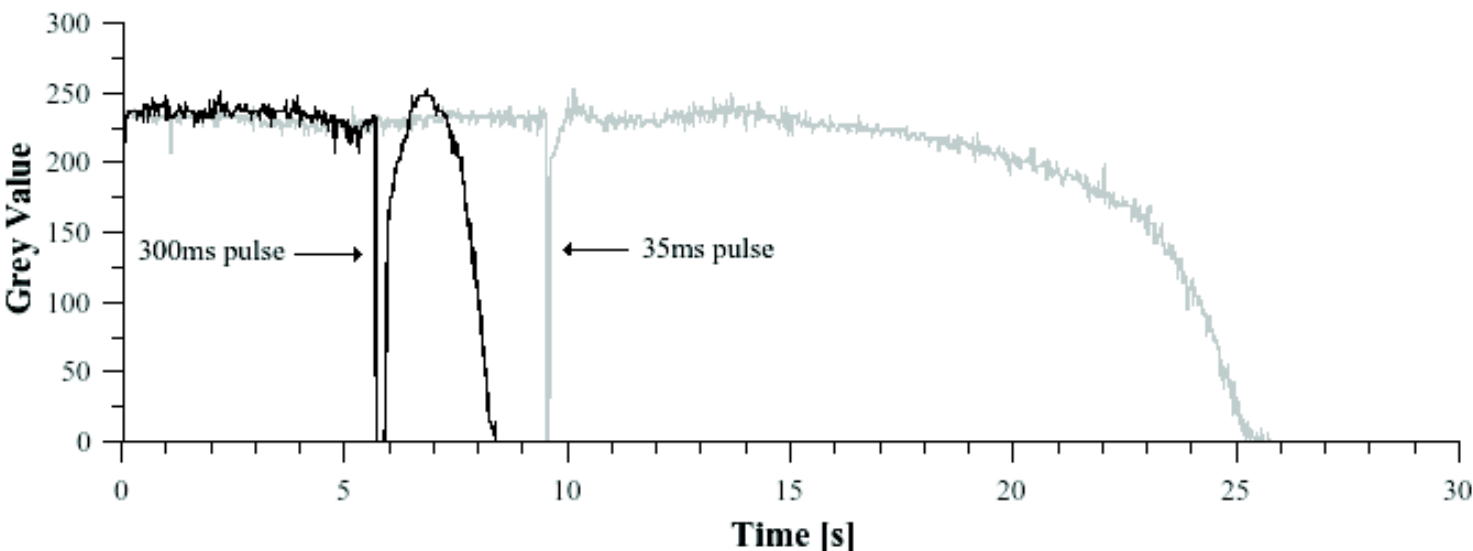

Figure 5. (A) Red blood cell and (B) Jurkat cell lysing (no flow) using a $35 \mathrm{~ms}$ light pulse. Scale bar: $20 \mu \mathrm{m}$. Pictures showing time lapse of lysis process, starting with 0s (before pulse), followed by snap-shots direct after the pulse. (C) Comparison of pulse times for red blood cell lysing. Change of the pixel intensities of the cell is recorded. Length of light pulse controls lysis time.

The results show that a short pulse is sufficient to induce lysis in different cell types. The time between the point of the treatment and the release of the cellular content is depending on the cell type. However, the time can be controlled by changing the pulse duration. In figure $5 \mathrm{C}$ a comparison between a $300 \mathrm{~ms}$ and $35 \mathrm{~ms}$ is illustrated. The pixel intensity of red blood cells is recorded over time and lysis is reached when cells are undistinguishable from the background of the image. When treated with a $300 \mathrm{~ms}$ pulse, lysis takes place within $2.5 \mathrm{~s}$ which is only one-tenth compared to a $35 \mathrm{~ms}$ pulse. This difference can be described with the pore formation process. The size and number of gaps in the membrane as well as the possibility of reversible pore formation and pore growth is triggered by the exposure time $e^{7,8}$. But not only the duration is improving the time of cell lysis, also an increase of the electric field strength by increasing the amplitude of the AC signal is in favour of faster cell disruption. It has to be noted that the experiments are conducted without fluid flow. An important factor like shear stress under laminar flow in microfluidic channels might influence the kinetic of electroporation, too.

In figure 6, an example of continuous cell lysis is shown. A combined use of OET and AT is applied to conduct the experiments. Blood cells $\left(10^{6} \mathrm{cells} / \mathrm{ml}\right)$ entering the microchannel and undergo acoustophoresis by an acoustic radiation force. This leads to a focused cell stream along the microchannel length. A light pattern is projected into the path of the concentrated cells. A continuous illumination is applied and the exposure time to induce cell lysis is controlled by the flow rate of the blood sample as well as the dimension of the light pattern. In figure 6A the ac field is switched off and only acoustic actuation is applied to the cells. When the nonuniform field is activated (figure 6B), approaching cells passing the illuminated area and disappear. It has to be noted that lysis is only induced and not complete after passing the exposure area. The disappearance is due to the dielectrophoretic force acting on the cells. Under the above mentioned 
experimental conditions, this force shows a negative sign which is pointing away from the high electric field strength. The result is a vertical displacement towards the top electrode which drives the cells out of focus. In the previous examples (batch mode), this effect is reduced by adsorption of the cell to the surface. The distance to the photoconductor surface can affect the lysis efficiency when the size of the light pattern is small. The induced transmembrane voltage to trigger pore formation decreases with distance from the high electric field strength. Therefore, it is important to control the illumination area to create a strong field across the channel height. Also, the stokes drag force should be higher than the dielectrophoretic force to avoid aggregations in front of the light pattern. Otherwise it can result in channel blockage and sample loss. Moreover, the acoustic radiation force is stronger than the electric force which keeps the cells aligned without significant lateral displacements.

(A)

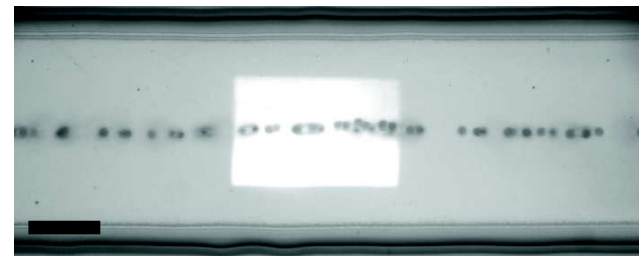

(B)

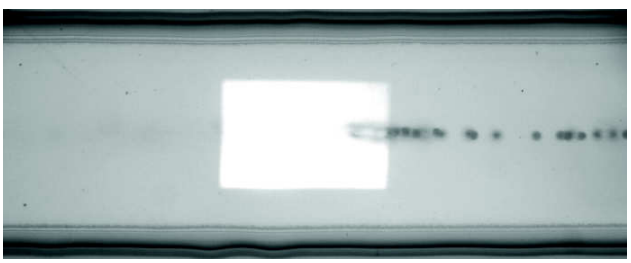

Figure 6. Blood cell lysing under continuous flow (flow from right to left). (A) Cells are concentrated along the pressure node by AT in the OET chip. Rectangular projection in the microchannel. This is the area where lysis is induced, when the electric field is switched on (B) The electric field is applied and the approaching blood cells disappear when passing the illuminated area. Here, a negative dielectrophoretic force is experienced by the cells which drives them out of focus. However, the short exposure $(300 \mathrm{~ms})$ while passing this area is sufficient to induce cell lysis. Scale bar: $50 \mu \mathrm{m}$.

The lysis efficiency of the continuous approach is quantified by collecting processed samples at the channel outlet. A flow rate of $3 \mu \mathrm{l} / \mathrm{min}$ is applied to the channel, $5 \mu \mathrm{l}$ samples are collected and diluted (1:2 or 1:5) for cell counting in a hemocytometer. In these experiments the illumination area is extended over the whole channel width to compare lysis efficiency when e.g. only the electric field (OET mode) is switched on. The length of the light pattern is $400 \mu \mathrm{m}$ which causes a exposure time of $75 \mathrm{~ms}$ under the applied flow rate. Furthermore, the influence of acoustic field on the cell count without electrical stimulation is investigated (AT mode). But first a control experiment is performed without electrical and acoustical actuation. Here, the sample is simply pumped through the microchannel and the determined cell concentration is compared to the original sample and the obtained cell concentration after the actuation of the chip. It is found that settling of cells in the connecting tubing occurs over time. Therefore the sampling process for each experiment is performed within 3 minutes to avoid incorrect measurements by sample loss in the tubing. Figure 7 gives an overview of the lysis efficiencies when the chip is actuated in the different modes. A blood cell concentration of $9 \cdot 10^{6}$ cells $/ \mathrm{ml}$ is used throughout this series of experiments. Without electrical or acoustical stimulation the control experiments (C, in figure 7) show no change in cell concentration as long as sampling time is kept small. Acoustic actuation only (AT mode), leads to a decrease in the cell population by $8 \%$. Applying an electric field under illumination (OET mode) drops the cell count by $48 \%$. The combined use of OET and AT results in a blood cell concentration of $0.4 \%$. Using both, acoustic and electric actuation, improves lysis significantly. Here, the concentration of the cell sample along the channel center is crucial to reach almost the entire cell population. Without acoustic focussing, cells are distributed over the channel width. But the channel walls are made of SU8 which act as insulator. When the electric field is switched on under illumination, the field strength is low in these region. Therefore, cells in proximity move towards the sidewalls. A lateral and vertical displacement is induced by the negative dielectrophoretic force. In total, the threshold membrane potential to induce electroporation is not obtained and only cells with a sufficient distance to the sides are lysed. The lateral movement is inhibited when the pressure node is created in the chip. Then, only a minor fraction of the population is not lysed. This might be attributed to larger cell aggregates or clumps which alter the field strength temporary. The exposure to ultrasound seems to have a slight effect on the overall cell count (8\%, figure 7). However, when blood cells are kept in a one dimensional trap without applying a fluid flow no morphological changes or lysis are observed over a time course of 45 minutes. The contribution of the pressure field to the lysis result can be neglected when working in the batch mode. But under continuous flow stokes drag combined with acoustic stimulation might increase the stress to the membrane and promotes cell lysis. 


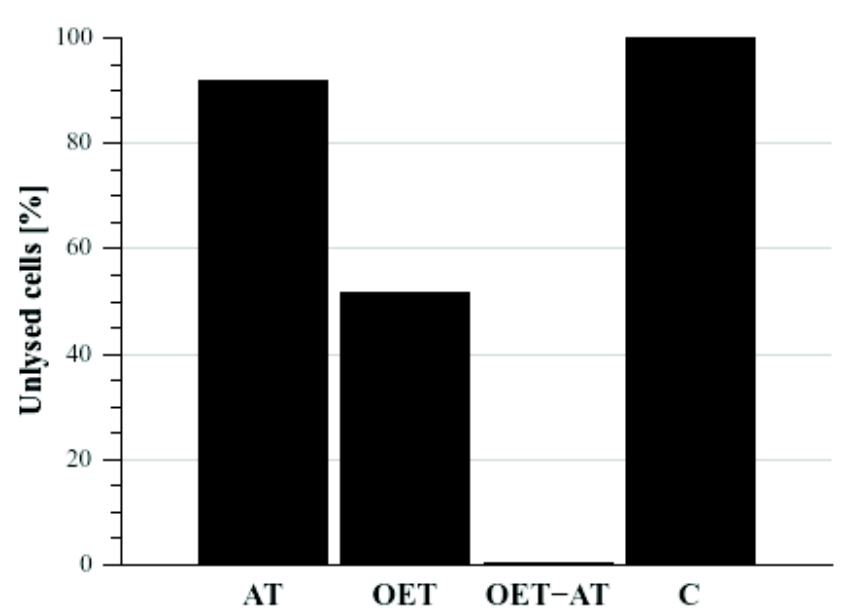

Figure 7. Quantification of unlysed blood cells under different conditions. AT $=$ only acoustic signal is applied. OET $=$ only electrical signal is applied. OET-AT = acoustic signal and electrical signal is applied. $\mathrm{C}=$ control and original cell concentration $\left(9 \cdot 10^{6} / \mathrm{ml}\right)$, cells are pumped through the channel without acoustic or electric actuation. $3 \mu \mathrm{l} / \mathrm{min}$ flow rate, $400 \mu \mathrm{m}$ light pattern, cells exposed for $\sim 75 \mathrm{~ms}$.

\section{CONCLUSION}

In summary, a system for cell lysis in a microfluidic environment is presented. Light induced electric fields are used to trigger electroporation on single cell level as well as lysis of concentrated blood samples. The size of the projected light pattern and the pulse duration control the lysis area and lysis time. A combination with acoustic tweezing through the use of an ultrasound transducer is demonstrated. This leads to concentration of cells in a one dimensional trap along the pressure node and improves irreversible electroporation under continuous flow. With the combined use of OET and AT, lysis efficiencies of $>99 \%$ are achieved.

\section{REFERENCES}

[1] Brown, R. B., Audet, J., “Current techniques for single-cell lysis,” J. R. Soc. Interface 5, 131-138 (2008).

[2] Kim, J., Johnson, M., Hill, P., Gale, B. K., "Microfluidic sample preparation: cell lysis and nucleic acid purification," Integrative Biology 1, 574-586 (2009).

[3] Quinto-Su, P. A., Lai, H., Yoon, H. H., Sims, C. E., Allbritton, N. L., Venugopalan, V., "Examination of laser microbeam cell lysis in a PDMS microfluidic channel using time-resolved imaging," Lab Chip 8, 408-414 (2008).

[4] Witte, C., Wilson, R., Cooper, J. M., Neale, S. L., “OET meets acoustic tweezing,” Proc. SPIE 8458 (2012).

[5] Menarchery, A., Kremer, C., Wong, P. E., Carlsson, A., Neale, S. L., Barrett, M. P., Cooper, J. M., "Counterflow Dielectrophoresis for Trypanosome Enrichment and Detection in Blood," Scientific Reports 2, 775 (2012).

[6] Lin, Y., Lee, G., “An optically induced cell lysis device using dielectrophoresis,” Appl. Phys. Lett. 94, 033901 (2009).

[7] Freeman, S. A., Wang, M. A., Weaver, J. C., "Theory of electroporation of planar bilayer membranes: predictions of the aqueous area, change in capacitance, and pore-pore separation," Biophys. J. 67, 42-56 (1994).

[8] Krassowska, W., Filev, P. D., "Modeling Electroporation in a Single Cell," Biophys. J. 92, 404-417 (2007). 